\title{
A Review of Ground Target Detection and Classification Techniques in Forward Scattering Radars
}

\author{
Mohammed E. A. Kanona \\ School of \\ Telecommunication \\ Future University \\ Khartoum, Sudan \\ mohammedkanona@gmail.com
}

\author{
Mohamed Ghazli Hamza \\ School of \\ Telecommunication \\ Future University \\ Khartoum, Sudan \\ mohghazli@gmail.com
}

\author{
Ashraf G. Abdalla \\ School of \\ Telecommunication \\ Future University \\ Khartoum, Sudan \\ agea33@yahoo.com
}

\author{
M. K. Hassan \\ Faculty of Electrical \\ Engineering \\ Universiti Teknologi \\ Malaysia, Johor, Malaysia \\ memo1023@gmail.com
}

\begin{abstract}
This paper presents a review of target detection and classification in forward scattering radar (FSR) which is a special state of bistatic radars, designed to detect and track moving targets in the narrow region along the transmitter-receiver base line. FSR has advantages and incredible features over other types of radar configurations. All previous studies proved that FSR can be used as an alternative system for ground target detection and classification. The radar and FSR fundamentals were addressed and classification algorithms and techniques were debated. On the other hand, the current and future applications and the limitations of FSR were discussed.
\end{abstract}

Keywords-neural network; PCA; Z-score; target classification; recognition; forward scattering radar

\section{INTRODUCTION}

Radar systems and stations are used for detecting various objects in space and establishing their current position, as well as determining velocities and trajectories for moving objects [1]. From the basic point of view, this is achieved by transmitting an electromagnetic (EM) wave from the transmitting antenna. If the target is located within the radar coverage area, the wave will be reflected back to the receiving antenna [2]. There are different types of radar systems, based on the transmitter-receiver topology: (a) the monostatic radar, where the transmitter and the receiver are spatially combined, and (b) the bistatic radar which consists of a single transmitter and a single receiver which are separated by a distance, comparable to the maximum range [1]. A Forward scattering radar (FSR) is a special state of bistatic radar [3], designed to detect and track targets moving in the narrow region along the transmitter-receiver base line. Its most attractive feature is radar cross section (RCS) which considers the target's physical cross section, the wavelength, the shape of the target's surface. A basic comparison to the traditional monostatic radar can be found in [4], whereas probably its most important feature is its robustness against stealth technology [5]. Moreover, the FSR receiver can utilize radiation from non-cooperative transmitters without revealing its location. In a hostile environment this is highly desirable as the receiver may be used covertly. All these advantage features created a 'come back' interest to FSR. In addition, FSR can be used for target classification, requires relatively simple hardware and has a long coherent interval of the received signal. This is the consequence of the loss in range resolution [6-7]. On the other hand, the FSR presents a conservative class of systems which have a number of fundamental limitations, that include the absence of the range resolution and operation within the narrow angles. This therefore requires the target to be very close to the transmitterreceiver baseline and the radar loses its ability to measure the range when the target crosses the base line.

\section{PREVIOUS AND RELATED WORKS}

Some of the main literature devoted to the FSR is given in $[8,9]$. Generally, there is a lack of recent publications on FSR. Earlier publications, pertaining to the forward scattering, were devoted to estimating the RCS of an object at the forward scattering. Among others, [5] provides a theoretical analysis and various experimental results to prove that the RAM coatings did not impose any effect on forward scattering when applied on highly conducting objects which were larger than the wavelength of the carrier. Nevertheless, the advantages of the FSR became known much later. These included the increase in the RCS of the object at the forward scattering. In [4], author presented a fast and simple approach for estimating the effective forward scattering RCS for the different targets at various operating frequencies. This was followed by [10], in which authors experimentally confirmed that the RCS, at forward scattering, was bigger than the one in the monostatic case by $30-40 \mathrm{~dB}$, depending on the frequency of the carrier. Authors in [11] discussed target detection and estimated the detection zones at forward scattering. They showed that the detection zone of a FSR was dependent on the type of the object and its flight trajectory. They calculated the bistatic RCS of the objects, related to the XY Cartesian co-ordinates to estimate the detection zones. Authors in [12] on the other hand, suggested that the detection is always lost at zero Doppler. This area is known as the 'dead zone'. Authors in [1] suggested that the areas worthy of investigation are the system issues of the 
FSR. These include system configurations and system parameters, such as the operating frequencies, power levels, and baseline distance. All that delayed the deployment of FSR in practical applications.

\section{A. Target Detection in FSR}

Authors in [13] discussed the FSR technology, the current and possible applications and the limitations of FSR in a feasibility study to the automatic ground target detection and classification. Also, the extraction of features from the radar measurements was introduced. Authors proved that the FSR system has a huge potential to be used as an alternative for ground target detection and classification based on PCA as feature extraction and the classification algorithm (k-nearest neighbor classifier) by a real experiment of three vehicles carried out on a public road. Authors in $[14,15]$ touched the problem of extracting the Doppler signature in different interference environments by using Hilbert transform and wavelet technique in order to predict the existence of target. Two experimentations have been done to collect the FSR signal under high clutter. The proposed method gave a good result with some reservations of wavelet issues. Soon after, authors in $[16,17]$ studied the effect of clutter on the automatic target classification (ATC) accuracy in FSR. It was shown that using conventional clutter-uncompensated ATC system can achieve high target classification accuracy at high SCR only, but the accuracy drops significantly with decreasing SCR. The employment of clutter-compensated ATC system is shown to improve significantly the classification accuracy at low SCR. Authors in [18] continued in the same field with new improvements in the existing method proposed in $[14,15]$ by considering a rough environment (receiver and surrounding noises). Results showed that target detection using a Hilbert transform is applicable only for certain conditions but target detection employing the wavelet technique is more robust against clutter and noise. An inclusive comparison of various wavelet threshold selection rules for different types of wavelet filters and levels of decomposition is conducted to study the effect on target detection with FSR. Two sets of field experiments were carried out to validate the proposed method, and target signals under the influence of large clutter were successfully detected using the proposed method with a confidence level exceeding $75 \%$. Then authors in [19] implemented the Haar and Meyer wavelet technique in FSR which gives more detailed scales and variation information from the measured signals. The results from the wavelet technique showed that they could find the similarity between signals of each target and dissimilarity between different targets.

Authors in [21] investigated accurate signal modeling for detecting moving targets in FSR by modifying the existing model algorithm by using numerous simulations. They claim that all the existing signal models and algorithms are built based on the assumptions that the baseline is long, diffraction angle is small and velocity direction of the target is approximately perpendicular to the baseline, the ground-based FSR system is characterized by short baseline and large diffraction angle and the velocity direction of the target is not always perpendicular to the baseline. Therefore in many cases, the above assumptions introduce significant errors to the results in the ground-based FSR. In the light of the ground-based FSR system, the signal model and imaging algorithm in traditional SISAR imaging technology are modified and gave good results. Authors in [22] state that, the received signal in FSR depends on the target's electrical size and trajectory, which are unknown a priori. As a result, in practical situations, it is impossible to obtain the accurate reference function at the reception side. That's why they proposed a signal processing algorithm which includes the construction of the adaptive reference functions and the identification of target velocity and observation time by the adaptation of an optimal filter (quasioptimal). They tested the algorithm performance under practical motion trajectories such as different motion directions and baseline crossing points, which indicates the effectiveness of the proposed algorithm in a practical case for FSR. As result they found that the proposed methods are suitable for the identification of target parameters, and in particular when observing target's time and speed. By knowing those parameters the possibility to obtain accurate target recognition rises.

Authors in [23] discussed FSR cross section of different target specification by conducting a simulation for analysis of a multi-car model. The study showed the effects of different target specifications on RCS radiation pattern at different angle for each frequency. Novel studies were in [24, 25]. Authors used GPS radio shadow instead of previous FSR models in order to build a passive FSR system. Investigation on different moving objects was introduced. The results showed that from FS-GPS radio shadows of different objects, information about the parameters of the object (size, speed and direction of movement, distance to the receiver) can be extracted from the width, shape and length of the received FS shadow. The occurrence of FS shadow is essential physical phenomena, which can be used to extract some useful information about the objects that create it. In [26], authors proved the forward scattering GPS radio shadows system can be used for detecting road vehicles in urban environment. Authors in [27] followed the previous study of target shadow with the practical aspects of the target profile reconstruction in a single node groundbased FSR system and discussed the target return signal for three different cars measured in real outdoors environment. The study proved that modeling approach can be successfully used for simulation of the Doppler phase history, which is required in the procedure of complex envelope extraction. The similarity of the reconstructed and original profiles demonstrates that TSPR delivers results suitable for both visual interpretations of target profile and ATC.

\section{B. Classification Techniques in FSR}

In [2, 28-30], it was shown that FSR can be effectively used for ground target detection, and in particular automatic vehicle recognition and classification using different techniques and scenarios. Researchers classified four different types of vehicles into categories based on their sizes and types at frequency $1 \mathrm{GHz}$ in ideal case scenario where the vehicles are crossing the baseline perpendicularly. In order to perform the classification, the combination of principal component analysis (PCA) and k-nearest neighbor (KNN) was proposed. Obtained 
results showed that good vehicle classification performance can be achieved. Authors in [31] worked on the evaluation of a network of forward scattering (FS) radar micro sensors for the detection and classification of ground targets based on the findings of [8]. The system power budget was operating in line of sight (LOS) conditions. It was evaluated at both theoretical and experimental level in terms of power budget analysis and resolution. Authors demonstrated that an excellent resolution is achievable. The potential resolution of the system is equal to the target's horizontal dimension. The dynamic range of the system is also shown to be very high. In addition, a number of practical targets were considered as simulation examples over a wide range of radar carrier frequencies. In [29], authors proved that FSR system has a huge potential to be used as an alternative system for ground target detection and classification. Authors in [32] proposed another solution based on using vehicle height and length and height profiles obtained by a microwave (MW) radar sensor. A precise feature vector can be extracted, and simple deterministic algorithms can be applied to determine the vehicle class. Field trials using a spread-spectrum MW radar sensor system operating on these principles have been carried out. They confirmed that accurate classification of a large number of vehicle classes can be reached.

Authors in [33] used image technique formulation to obtain the electric field integral equations (EFIEs) in order to classify cylindrical targets from their ultra wide-band radar returns. Then, the EFIEs were solved numerically by the method of moment (MoM). Because of the wide frequency range of the ultrawide-band radar signal, the database to be used for target classification becomes very large. To deal with this problem and to provide robustness, wavelet transform was utilized. Application of wavelet transform significantly reduces the size of the database. The coefficients obtained by wavelet transform are used as the inputs of artificial neural networks (ANNs). Then, the actual performances of the ANNs were investigated by receiver operating characteristic (ROC) analysis. Therefore, the compressed inputs of the ANNs were determined and a dataset was formed. RBF, GRNN and MLP were investigated in this work. According to the testing and training rates, the best classifier is the MLP. To support this result, sensitivity and specificity values and the ROC curves were obtained and it was observed that MLP was better. Authors in dealt with a new system that uses neural network(NN)-based methodology with various types of training algorithms. It reiterates the uniqueness and the ability of the neural network implementation to accurately classify unknown vehicle signature on the available training data, the input of $\mathrm{NN}$ is defined as the vehicle length and back-propagation NN (BPNN) was used as a NN model. The paper proves that $\mathrm{NN}$ is suitable to be used as a classifier since the classification accuracy exceeds more than $90 \%$. Authors in [35] on the other hand, proved the potential and utilization of NNs by comparing the KNN classifier and the conventional method (PCA) with the proposed. Results showed that NNs can be effectively employed in FSR as an automatic classifier. After implementing multi-layer perceptron (MLP), a BPNN trained with three back-propagation algorithms gave very promising results in vehicle recognition and vehicle categorization. $10 \%$ of overall data was misclassified in vehicle recognition and only $2 \%$ of overall data was misclassified in vehicle categorization. The same classification method was applied in $[14,36]$, but with different trajectory known as angle of detection. Theoretically, target's trajectory is one of the factors affecting the target's signature which contributes towards the poor performance of the classification system. Hence, by using multiple sensors, the discrepancies in classification performance could be reduced. Later, the same classification system has been tested at low frequencies: Ultra High Frequency (UHF) and Very High Frequency (VHF) bands [37]. The paper proved that a good classification performance can be obtained even at low frequency.

Authors in [38] proposed a novel ground vehicle classification approach using unmodulated $\mathrm{CW}$ radar. The radar was set up to look forward down to the road. Vehicles were modeled as body targets composed of multiple scattering centers. Analysis showed that the spatial distribution of scattering centers can be derived from the Doppler signature of radar echo. Hough transform was performed to estimate the distribution which was then used for classification. In experiments, vehicles were classified into three types at an average accuracy of $94.8 \%$. Authors in $[39,40]$ designed and developed three novel, distinct automatic target recognition (ATR) methods. For the classification they divided the observed targets into predefined classes (extremely randomized trees or subspace methods). A key feature of the approach was the breaking of the recognition problem into a set of subproblems by decomposing the parameter space, which consist of the frequency, the polarization, the aspect angle and the bistatic angle, into regions and build one recognizer for each region. Authors in [41] claimed that all previous studies did not consider a rough environment analysis in ground target classification systems and all experiments have been on ideal environment which significantly effects the classifier output. After adding simulation noises to the FSR signal output NN was used as classifier. As result it was found that classification using an ANN is robust against noise to a certain extent of noise. They developed an enhanced classification process in [42]. However the performance of the classification system was still below satisfactory level especially under the influence of external factors such as clutter [16], target trajectory uncertainties [43] and features used as the input to the classifier.

Authors in [44] addressed the importance of feature extraction process in the FSR system by evaluating manual and automatic reduction techniques (PCA and Z-score). The main objective of this study was to analyze the most suitable feature extraction algorithm to classify ground vehicles based on their physical size. They continued in $[45,46]$ by improving the classification accuracy by the combination of Z-score and $\mathrm{NN}$. It was shown that as the number of features increases, the classification accuracy increases. The highest percentage of classification accuracy can be achieved when using a NN5 system. Authors in [47] used LTE signal as a source for passive bistatic radar (PBR) for detection and location of ground moving targets depending on bistatic RCS. Conventional processing was used as classification approach. They performed simulations using computer simulation technology (CST) microwave studio. The simulation results showed that 
the large area of ground moving target, had better outcome compared to other ground moving targets which is compatible with Babinet's principle, which declares a target of physical cross-sectional area is proportionate to RCS. In [48], same authors rolled in their previous study but this time detected humans instead of vehicles. Real experiments were done by testing and evaluating different human sizes. It was discovered that the PSD of the individuals are inversely proportional to their heights. In PCA, data of the individuals show a good convergence in terms of their respective groups. Authors in $[49,50]$ proposed a passive FSR system that can exploit the peculiar advantages of the enhancement in forward scatter radar cross section (FSRCS) for target detection and recognition using LTE signals. This paper illustrates the first classification result in the passive FSR system. The great potential in the passive FSR system provides a new research area in passive radar that can be used for diverse remote monitoring applications. In [51], authors presented the latest feasibility studies and experimental results from using LTE signals in PBR applications. Details are provided about aspects such as signal characteristics, experimental configurations, and SNR studies. Six experimental scenarios were carried out to investigate the detection performance of the proposed system on ground-moving targets. The detection ability was demonstrated through the use of a cross-ambiguity function. The detection results suggested that LTE signals are suitable as a source signal for PBR.

\section{CONCLUSION}

This paper presents a review of target detection and classification in FSR and its advantages over other types of radar configuration. It is generally accepted that FSR can be used as an alternative system for ground target detection and classification. Further, recent research focused on the application of different classifiers to accurately classify unknown vehicle signatures. Moreover, feature extraction was addressed, especially PCA and Z-score to improve classification accuracy. The area of ground vehicle classification is very interesting, that's why intensive studies have been conducted in the last few years looking for alternatives to old systems, using FSR theory to reduce the cost and to make use of the existing transmitted signals. Therefore, GSM, LTE, GPS and FM were tested for detection and recognition of vehicles and humans as well using the same classification techniques. However, a number of problems are still unsolved, including the choice of optimum frequency, a more precise and intelligent speed estimation algorithm plus the absence of the range resolution and operation within the narrow angles. Another set of problems is the choice of the feature extraction before injecting the signature into the classifier. Future research will focus on using artificial intelligence, especially NNs with bigger databases and feature extraction techniques as pre-processing to improve the classification system in order to implement automatic classification, in order to lead to wider use of FSR in other areas.

\section{REFERENCES}

[1] M. Cherniakov (ed), Bistatic radar: principles and practice, Wiley, 2007
[2] A. R. S. A. Raja, Forward scattering radar for vehicle classification, $\mathrm{PhD}$ Thesis, University of Birmingham, 2007

[3] M. I. Skolnik, Introduction to Radar Systems, McGraw-Hill, NY,USA, 1962

[4] J. L. Glaser, "Bistatic RCS of complex objects near forward scatter. IEEE transactions on aerospace and electronic systems", Vol. AES-21, No. 1, pp. 70-78, 1985

[5] R. E. Hiatt, K. M. Siegel, H. Weil, "Forward scattering by coated objects illuminated by short wavelength radar", Proceedings of the IRE, Vol. 48, No. 9, pp. 1630-1635, 1960

[6] H. Sun, D. K. P. Tan, Y. Lu, "Design and implementation of an experimental GSM based passive radar", Proceedings of the International Radar Conference, Adelaide, Australia IEEE, 2003

[7] D. K. P. Tan, H. Sun, Y. Lu, "Sea and air moving target measurements using a GSM based passive radar", IEEE International Radar Conference, Arlington, USA, IEEE, 2005

[8] M. Cherniakov, V. V. Chapurskiy, R. R. Abdullah, P. Jancovic, M. Salous, "Short-range forward scattering radar", International Radar Conference, pp. 322-328, 2004

[9] M. I. Skolnik, Radar handbook, McGraw Hill Professional, 1970

[10] Y. S. Chesnokov, M. V. Krutikov, "Bistatic RCS of aircrafts at the forward scattering. in Radar", CIE International Conference of Radar, Beijing, China, IEEE, 1996

[11] A. B. Blyakhman, A. G. Ryndyk, S. B. Sidorov, "Forward scattering radar moving object coordinate measurement", The Record of the IEEE 2000 International Radar Conference, Alexandria, VA, USA, IEEE, 2000

[12] D. M. Gould, R. S. Orton, R. J. E. Pollard, Forward scatter radar detection, in RADAR 2002, Edinburgh, UK, pp. 36-40, 2002

[13] R. Abdullah, A. Ismail, "Forward scattering radar current and future application", International Journal of Engineering and Technology, Vol. 3, No. 1, pp. 61-67, 2006

[14] K. H. Mohamed, M. Cherniakov, M. F. A. Rasid, R. S. A. Raja Abdullah, "Automatic target detection using wavelet technique in forward scattering radar", in EuRAD 2008, European Radar Conference, Amsterdam, Netherlands, October 30-31, 2008

[15] M. K. H. M. Alla, R. S. A. Raja Abdullah, M. F. A. Raseed, "Detection of ground target in forward scattering RADAR using Hilbert transform and wavelet technique", International Review of Electrical Engineering, Vol. 4, No. 2, pp. 320-326, 2009

[16] N. E. Abd Rashid, P. Jancovic, M. Gashinova, M. Cherniakov, V. Sizov, "The effect of clutter on the automatic target classification accuracy in FSR", in 2010 IEEE Radar Conference, Washington, DC, USA, IEEE, 2010

[17] N. E. B. Abd Rashid, "Automatic vehicle classification in a low frequency forward scatter micro-radar", $\mathrm{PhD}$ Thesis, University of Birmingham, 2012

[18] R. S. A. Raja Abdullah, M. F. A. Rasid, M. K. Mohamed, "Improvement in detection with forward scattering radar", Science China Information Sciences, Vol. 54, No. 12, pp. 2660-2672, 2011

[19] K. A. Othman, M. I. Jusoh, N. E. Abd Rashid, C. W. F. C. Wan Fadhil, "Wavelet Technique Implementation in Forward Scattering Radar (FSR) Ground Target Signal Processing", Journal of Telecommunication, Electronic and Computer Engineering, Vol. 9, No. 1-5, pp. 59-62, 2017

[20] M. Salah, M. F. A. Rasid, R. S. A. Raja Abdullah, M. Cherniakov, "Speed estimation in forward scattering radar by using standard deviation method", Modern Applied Science, Vol. 3, No. 3, pp. 16-25, 2009

[21] T. Zeng, X. Li, C. Hu, T. Long, "Investigation on accurate signal modelling and imaging of the moving target in ground-based forward scatter radar", IET Radar, Sonar \& Navigation, Vol. 5, No. 8, pp. 862870, 2011

[22] C. Hu, V. Sizov, M. Anoniou, M. Gashinova, M. Cherniakov, "Optimal signal processing in ground-based forward scatter micro radars", IEEE Transactions on Aerospace and Electronic Systems, Vol. 48, No. 4, pp. 3006-3026, 2012 
[23] N. A. M. Daud, N. E. Abd Rashid, K. A. Othman, N. Ahmad, "Analysis on Radar Cross Section of different target specifications for Forward Scatter Radar (FSR)", in Fourth International Conference on Digital Information and Communication Technology and it's Applications, Bangkok, Thailand, IEEE, 2014

[24] C. Kabakchiev, I. Garvanov, V. Behar, P. Daskalov, H. Rohling, "Study of moving target shadows using passive Forward Scatter radar systems", in 15th International Radar Symposium, Gdansk, Poland, IEEE, 2014

[25] C. Kabakchiev, K. Kabakchiev, I. Garvanov, V. Behar, K. Kulpa, H. Rohling, D. Kabakchieva, A. Yarovoy, "Experimental verification of target shadow parameter estimation", in 17th International Radar Symposium, Krakow, Poland, IEEEm 2016

[26] I. Garvanov, C. Kabakchiev, V. Behar, M. Garvanova, "Target detection using a GPS Forward-Scattering Radar", in International Conference on Engineering and Telecommunication, Moscow, Russia, IEEE, 2015

[27] S. Hristov, L. Daniel, E. Hoare, M. Cherniakov, M. Gashinova, "Target shadow profile reconstruction in ground-based forward scatter radar", in 2015 IEEE Radar Conference, Arlington, VA, USA, IEEE, 2015

[28] F. Kruse, F. Folster, M. Ahrholdt, H. Rohling, M. M. Meinecke, T. B. To "Target classification based on near-distance radar sensors", in 2004 IEEE Intelligent Vehicles Symposium, Parma, Italy, IEEE, pp. 722-727, 2004

[29] M. Cherniakov, M. Salous, R. S. A. Raja Abdullah, V. Kostylev, "Forward scattering radar for ground targets detection and recognition", in Defence Technology Conference, Edinburgh, UK. 2005

[30] M. Cherniakov, R. S. A. R. Abdullah, P. Jancovic, M. Salous, V. Chapursky, "Automatic ground target classification using forward scattering radar", IEE Proceedings-Radar, Sonar and Navigation, Vol. 153, No. 5, pp. 427-437, 2006

[31] M. Cherniakov, M. Salous, V. Kostylev, R. S. A. Raja Abdullah, "Analysis of Forward Scattering Radar for Ground Target Detection", in 2005 European Radar Conference, Paris, France, pp. 165-168, IEEE, 2005

[32] I. Urazghildiiev, R. Ragnarsson, P. Ridderstrom, A. Rydberg, E. Ojefors, K. Wallin, P. Enochsson, M. Ericson, G. Lofqvist, "Vehicle classification based on the radar measurement of height profiles", IEEE Transactions on Intelligent Transportation Systems, Vol. 8, No. 2, pp. 245-253, 2007

[33] S. Makal, A. Kizilay, L. Durak, "On the target classification through wavelet-compressed scattered ultrawide-band electric field data and ROC analysis", Progress In Electromagnetics Research, Vol. 82, pp. 419-431, 2008

[34] R. S. A. Raja Abdullah, M. I. Saripan, M. Cherniakov, "Neural network based for automatic vehicle classification in forward scattering radar", I n 2007 IET International Conference on Radar Systems, Edinburgh, UK, IEEE, 2007

[35] N. K. Ibrahim, R. S. A. Raja Abdullah, M. I. Saripan, "Artificial neural network approach in radar target classification", Journal of Computer Science, Vol. 5, No. 1, pp. 23-32, 2009

[36] M. Cherniakov, R. S. A. Raja Abdullah, P. Jancovic, M. Salous, "Forward scattering micro sensor for vehicle classification", in 2005 IEEE International Radar Conference, Arlington, VA, USA, IEEE, 2005

[37] N. E. A. Rashid, M. Antoniou, P. Jancovic, V. Sizov, R. Abdullah, M. Cherniakov, "Automatic target classification in a low frequency FSR network", in European Radar Conference, Amsterdam, Netherlands, IEEE, 2008

[38] J. X. Fang, H. D. Meng, H. Zhang, X. Q. Wang, "A ground vehicle classification approach using unmodulated continuous-wave radar", in 2007 IET International Conference on Radar Systems, Edinburgh, UK, IEEE 2007

[39] J. Pisane, Automatic target recognition using passive bistatic radar signals, PhD Thesis, Supélec, 2013

[40] J. Pisane, S. Azarian, M. Lesturgie, J. Verly, "Automatic target recognition for passive radar", IEEE Transactions on Aerospace and Electronic Systems, Vol. 50, No. 1, pp. 371-392, 2014

[41] M. K. H. M. Alla, M. Kanona, A. G. Elsid, "Target Classification in Forward Scattering Radar in Noisy Environment”, International Journal of Application or Innovation in Engineering \& Management, Vol. 3, No. 11 , pp. 1-5, 2014

[42] M. E. A. Kanona, A. G. Abdalla, M. K. H. M. Alla, Y. A. Hamdalla, "Enhanced Neural Network Based Ground Target Classification", Technology Horizons Journal, Vol. 1, pp. 1-5, 2018

[43] N. E. A Rashid, N. Ahmad, N. F. Abdullah Nor, N. Ismail, A. A. Bt Jamaludin, "The effect of different ground characteristic to the stability and similarity of target spectra in FSR micro-sensor network", in 2012 IEEE Student Conference on Research and Development, Pulau Pinang, Malaysia, IEEE, 2012

[44] N. F. Abdullah, N. E. A. Rashid, I. Musirin, Z. I. Khan, "Vehicles classification based on different combination of feature extraction algorithm with neural network (nn) using forward scattering radar (FSR)", Journal of Theoretical \& Applied Information Technology,. Vol. 77, No. 3, pp. 311-317, 2015

[45] N. F. Abdullah, N. E. A. Rashid, K. A. Othman, Z. I. Khan, I. Musirin, "Ground Vehicles Classification using Multi Perspective Features in FSR Micro-Sensor Network", Journal of Telecommunication, Electronic and Computer Engineering, Vol. 9, No. 1-5, pp. 49-52, 2017

[46] N. F. Abdullah, N. E. A. Rashid, Z. I. Khan, I. Musirin, "Analysis of different Z-score data to the neural network for automatic FSR vehicle classification", IET International Radar Conference, Hangzhou, China,IEEE, 2015

[47] N. A. Aziz, R. S. A. R. Abdullah, "RCS classification on ground moving target using lte passive bistatic radar", Journal of Scientific Research and Development, Vol. 3, No.2. pp. 57-61, 2016

[48] N. H. A. Aziz, R. S. A. R. Abdullah, A. N. M. Yusof, "Human Detection and Recognition System Using Passive Forward Scattering Radar", Science International, Vol. 29, No. 1, pp. 69-73, 2017

[49] R. S. A. R. Abdullah, N. H. A. Aziz, N. E. Abdul Rashid, A. A. Salah, F. Hashmin, "Analysis on Target Detection and Classification in LTE Based Passive Forward Scattering Radar", Sensors, Vol. 16, No. 10, p. 1607, 2016

[50] R. S. A. R. Abdullah, A. A. Salah, N. H. A. Aziz, N. E. Abdul Rashid, "Vehicle recognition analysis in LTE based forward scattering radar", in IEEE Radar Conference, Philadelphia, PA, USA, IEEE, 2016

[51] R. S. A. R. Abdullah, A. A. Salah, A. Ismail, F. Hashim, N. E. Abdul Rashid, N. H. A. Aziz, "LTE-Based Passive Bistatic Radar System for Detection of Ground-Moving Targets", ETRI Journal, Vol. 38, No. 2, pp. 302-313, 2016 